\title{
Side milling of ruled surfaces: Optimum positioning of the milling cutter and calculation of interference
}

\author{
Jean-Max REDONNET, Walter RUBIO and Gilles DESSEIN \\ Université de Toulouse, Institut Clément Ader \\ UPS, 118 rte de Narbonne, 31062 Toulouse France \\ International Journal of Advanced Manufacturing Technology, vol 14 (7), pp 459-465, 1998
}

DOI: $10.1007 /$ BF01351391

\begin{abstract}
Side milling is a process that enables machining time and thus costs, to be reduced. This type of machining is particularly well suited to ruled surfaces and all surfaces where one of the principal curvatures is very small compared to the tool radius and changes little oiler the entire surface. These surfaces must be treated with great care, as they are often on parts with high added value such as turbine blades, aircraft wings or helicoidal parts as used in fluid dynamics.

We then need to calculate and minimise interference that may arise if the I ruled surface cannot be developed. Whereas machining is usually carried out by setting the tool according to a rule, we suggest a new setting of the tool allowing interference to be reduced considerably. The computation algorithms for this setting were developed so as to be used in real-time by CAD/CAM software.

A comparative study of errors made with each type of setting is also presented. This shows a considerable reduction in errors when the setting we suggest is implemented.
\end{abstract}

Keywords: five-axis machines; interferences; NURBS; ruled surfaces; side milling

\section{Introduction}

Side milling has been less frequently addressed by researchers than end milling (see [1-4]), but it may be very useful, especially for ruled surfaces. We shall first briefly recapitulate the principle for ruled surfaces and settings that are currently adopted in existing CAD/CAM software before going on to introduce a new setting for the tool, allowing interference to be reduced considerably. The second part of the article is devoted to determining the parameters for this setting. We shall then make a comparative analysis based on the calculation of interference for each type of setting.

\subsection{Ruled Surfaces}

A ruled surface is generated by a set of straight lines based on two directrices.

The equation corresponding to the ruled surface in
Fig. 1 is:

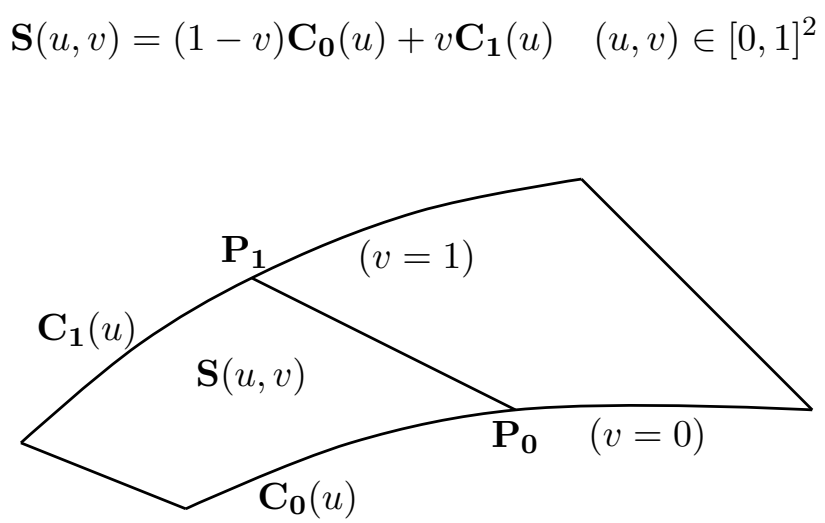

Figure 1: Ruled surface

\section{2 "Standard" setting}

With "standard" setting the tool axis is collinear with the rule under consideration. The tool can then 


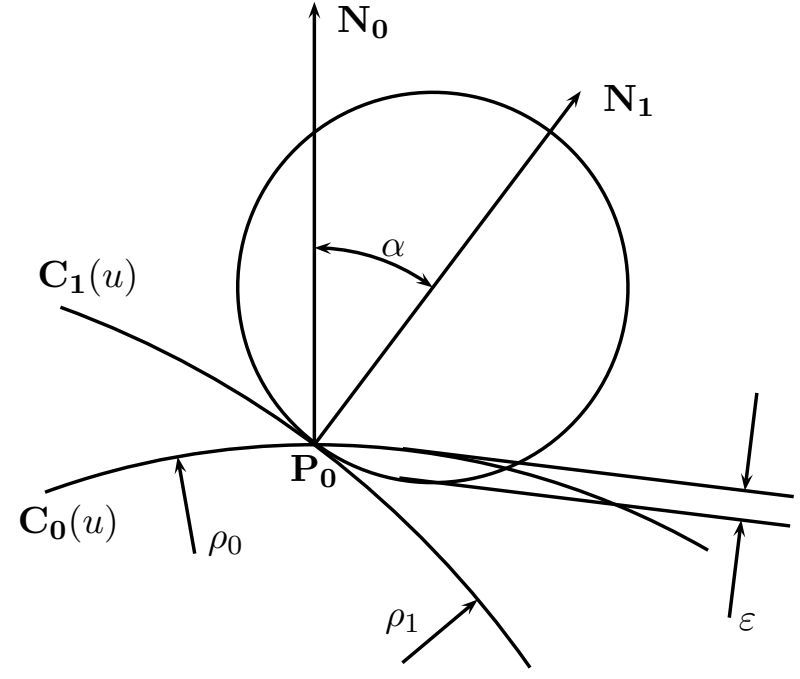

(a)

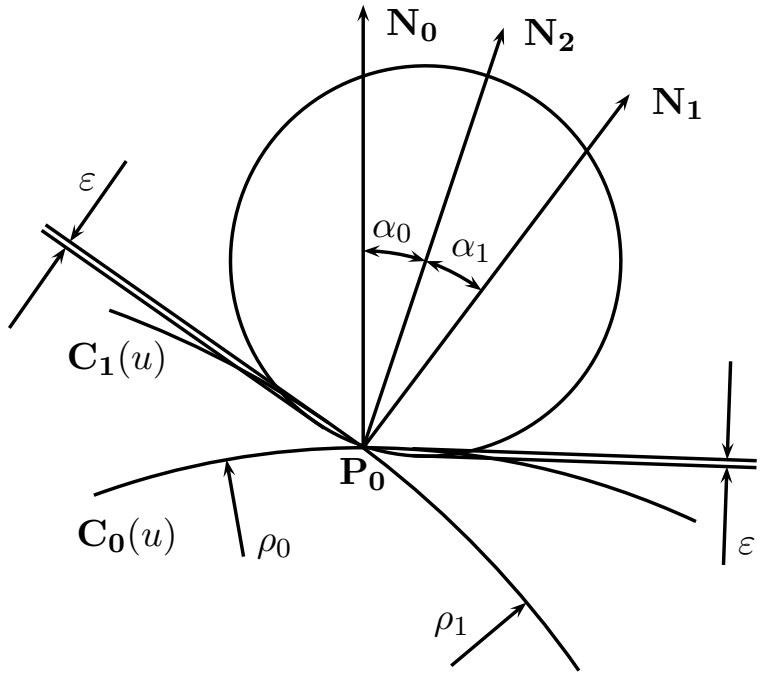

(b)

Figure 2: "Standard" tool setting.

be placed at a tangent to one of the two directrices (Fig. 2a) or so as to distribute the error between its two extremities (solution proposed by Rubio $[5,6]$; Fig. 2b).

According to [5], we then obtain in case (a):

$$
\varepsilon=\rho_{1}+R-\sqrt{R^{2}+\rho_{1}^{2}+2 R \rho_{1} \cos (\alpha)}
$$

in case (b):

$$
\begin{aligned}
\varepsilon & =\rho_{0}+R-\sqrt{R^{2}+\rho_{0}^{2}+2 R \rho_{0} \cos \left(\alpha_{0}\right)} \\
& =\rho_{1}+R-\sqrt{R^{2}+\rho_{1}^{2}+2 R \rho_{1} \cos \left(\alpha_{1}\right)}
\end{aligned}
$$

These equations can be used to determine the tool radius $R$ needed to respect a given tolerance. In the case of infinite curvature radii (straight directrices), error calculation for the standard setting will give (see $[6])$ :

in case $(\mathrm{a})$ :

$$
\varepsilon=R(1-\cos (\alpha))
$$

in case (b):

$$
\varepsilon=R\left(1-\cos \left(\alpha_{0}\right)\right)
$$

\section{$1.3 \quad$ Optimum Setting}

The setting we suggest is the fruit of a trade-off between two contradictory objectives:

1. To reduce interference by a more complex tool setting that therefore takes longer to calculate than the standard setting.
2. To be able to calculate this setting rapidly enough to enable real-time utilisation in CAD/CAM type software.

This optimum setting as described in Fig. 3 is based on 3 tangential points:

- Tangency of the tool lower generating line to the rule $\mathbf{P}_{\mathbf{0}} \mathbf{P}_{\mathbf{1}}$ : point $\mathbf{M}_{\mathbf{2}}$.

- Tangency to the two directrices $\mathbf{C}_{0}(u)$ and $\mathbf{C}_{\mathbf{1}}(u)$ on both sides of the extreme points of the rule: points $\mathbf{M}_{\mathbf{0}}$ and $\mathbf{M}_{\mathbf{1}}$.

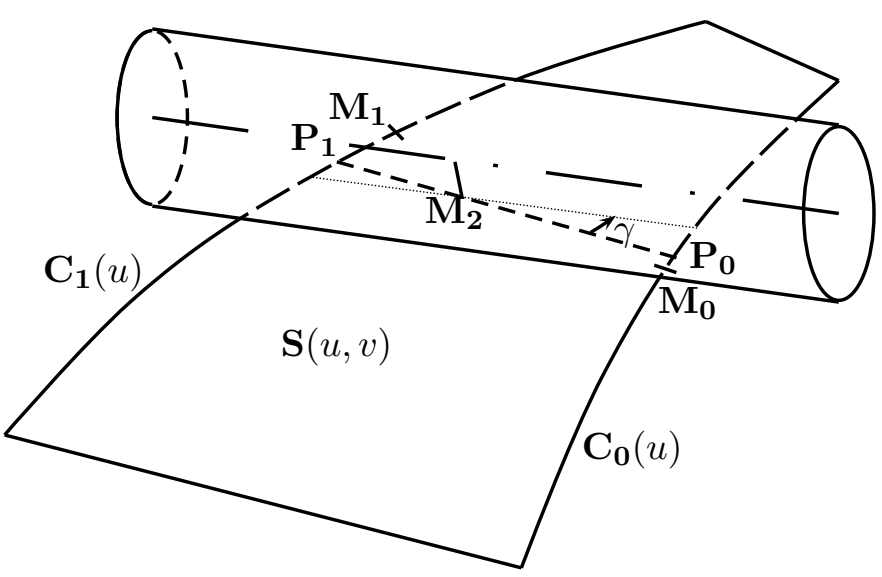

Figure 3: Optimum setting

Marciniak first proposed that the tool may be shifted along the rule (see [1] pp. 233,234), but the extra degree of freedom introduced in this way needs an additional drive surface to be eliminated. In [7], Liu presents a method based on two offset points (the 
double point offset method) introducing an angle between the rule and the tool axis. In Section 4.2 we shall compare the results obtained using each method for the test surface presented in this paper.

With our setting, the tool position can thus be completely defined by two parameters: the angle $\gamma$ made between the tool axis and the rule, and the position of the point $\mathbf{M}_{\mathbf{2}}$ on the rule $\mathbf{P}_{\mathbf{0}} \mathbf{P}_{\mathbf{1}}$ (once the direction of the rotation axis is established).

\section{Determining Setting Parameters}

\subsection{Preliminary Calculations}

We first carry out the following preliminary calculations [8-10]: The position of the rule is defined by $u=u_{p}$. We then consider the vectors $\mathbf{T}_{\mathbf{0}}$ and $\mathbf{T}_{\mathbf{1}}$ tangent to the directrices $\mathbf{C}_{\mathbf{0}}(u)$ and $\mathbf{C}_{\mathbf{1}}(u)$ at the extremities of the rule $\mathbf{P}_{\mathbf{0}}$ and $\mathbf{P}_{\mathbf{1}}$, and the vectors $\mathbf{N}_{\mathbf{0}}$ and $\mathbf{N}_{\mathbf{1}}$ perpendicular to the directrices $\mathbf{C}_{\mathbf{0}}(u)$ and $\mathbf{C}_{\mathbf{1}}(u)$ at $\mathbf{P}_{\mathbf{0}}$ and $\mathbf{P}_{\mathbf{1}}$ and also perpendicular to the rule $\mathbf{P}_{\mathbf{0}} \mathbf{P}_{\mathbf{1}}$, such that

$$
\mathbf{N}_{0}=\mathbf{T}_{0} \times \mathbf{P}_{0} \mathbf{P}_{1} \quad \text { and } \quad \mathbf{N}_{1}=\mathbf{T}_{1} \times \mathbf{P}_{\mathbf{0}} \mathbf{P}_{1}
$$

We establish

$$
\begin{aligned}
& R=\text { tool radius } \\
& h_{p}=\text { length of the rule at position } u_{p} \\
& h_{0}=\text { distance } \mathbf{P}_{\mathbf{0}}, \mathbf{M}_{\mathbf{2}} \\
& h_{1}=\text { distance } \mathbf{P}_{\mathbf{1}}, \mathbf{M}_{\mathbf{2}}
\end{aligned}
$$

and obtain $h_{0}+h_{1}=h_{p}$

We then define the following references (see Fig. 4):

1. Reference $\mathcal{R}_{1}$ related to the rule:

- origin $\mathbf{P}_{2}$ : at the centre of the rule

- vector $\mathbf{z}_{1}$ : along the direction of the rule

- vector $\mathbf{y}_{\mathbf{1}}$ : along the direction of the bisector of the angle formed by the projections of $\mathbf{N}_{\mathbf{0}}$ and $\mathbf{N}_{\mathbf{1}}$ in the plane perpendicular to $\mathbf{z}_{\mathbf{1}}$ going through $\mathbf{P}_{\mathbf{2}}$ (vector $\mathbf{N}_{\mathbf{2}}$ in Fig. $2 \mathrm{~b}$ with $\alpha_{0}=\alpha_{1}$ )

- vector $\mathrm{x}_{1}$ : result of the vector product between $\mathbf{y}_{1}$ and $\mathbf{z}_{1}$

We can then calculate the matrices for passage between the overall reference in which the geometrical entities are defined and $\mathcal{R}_{1}$.

2. Reference $\mathcal{R}_{2}$ related to the tool:

- origin $\mathbf{P}_{2}$ : tangential point of the tool on the rule

- vector $\mathbf{y}_{\mathbf{2}}$ : equal to $\mathbf{y}_{\mathbf{1}}$
- vector $\mathbf{z}_{2}$ : projection of the tool axis on the plane $\mathbf{P}_{\mathbf{2}}, \mathbf{z}_{1}, \mathbf{x}_{\mathbf{1}}$

- vector $\mathbf{x}_{2}$ : result of the vector product between $\mathbf{y}_{\mathbf{2}}$ and $\mathbf{z}_{\mathbf{2}}$

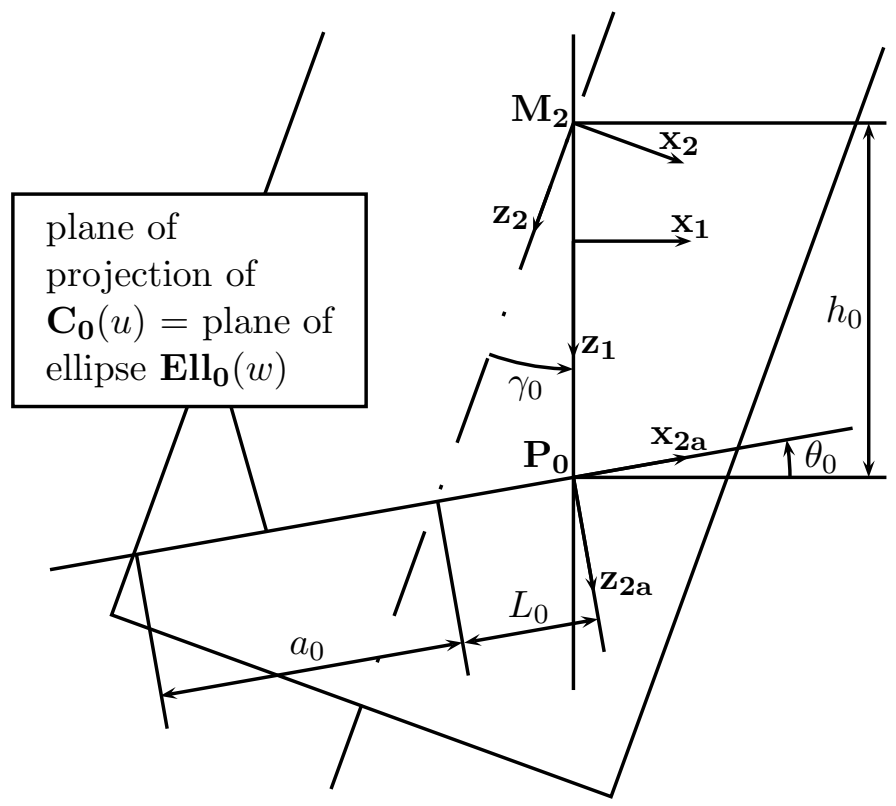

view along direction $\mathbf{y}_{\mathbf{1}}=\mathbf{y}_{\mathbf{2}}=\mathbf{y}_{\mathbf{2 a}}=\mathbf{y}_{\mathbf{2 b}}$

Figure 4: Definition of references.

In this reference, the equation for the tool will be:

$$
\mathbf{F}(s, t)=\left(\begin{array}{c}
R \cos (s) \\
R(\sin (s)+1) \\
t
\end{array}\right)
$$

with $s \in[0,2 \pi]$ and $t \in\left[-\frac{h}{2}, \frac{h}{2}\right]$

where $h$ is large enough to cover the entire desired length $\left(h>h_{p} \cos (\gamma)\right)$.

Rotation between $\mathcal{R}_{1}$ and $\mathcal{R}_{2}$ will then be $\gamma$. Translation between $\mathcal{R}_{1}$ and $\mathcal{R}_{2}$ will be given by $h_{p} / 2-h_{o}$.

3. Reference $\mathcal{R}_{2 a}$ related to $\mathbf{P}_{\mathbf{0}}$ :

- origin $\mathbf{P}_{\mathbf{0}}$

- vector $\mathbf{y}_{\mathbf{2}}$ : equal to $\mathbf{y}_{\mathbf{1}}$

- vector $\mathbf{x}_{\mathbf{2 a}}$ : perpendicular to $\mathbf{y}_{\mathbf{2 a}}$ and contained in the plane defined by $\mathbf{y}_{\mathbf{2 a}}$ and $\mathbf{C}_{\mathbf{0}}(u)$

- vector $\mathbf{z}_{\mathbf{2 a}}$ : result of the vector product between $\mathbf{x}_{\mathbf{2}} \mathbf{a}$ and $\mathbf{y}_{\mathbf{2} \mathbf{a}}$.

4. Reference $\mathcal{R}_{2 b}$ related to $\mathbf{P}_{\mathbf{1}}$ : defined as $\mathcal{R}_{2 a}$ for $\mathbf{P}_{1}$ and $\mathbf{C}_{1}(u)$ 
Rotation between references $\mathcal{R}_{2 a}$ and $\mathcal{R}_{1}$ will be given by

$$
\theta_{0}=\arctan \left(\frac{\mathbf{T}_{\mathbf{0}} \cdot \mathbf{z}_{\mathbf{1}}}{\mathbf{T}_{\mathbf{0}} \cdot \mathbf{x}_{\mathbf{1}}}\right)
$$

Similarly, between $\mathcal{R}_{2 b}$ and $\mathcal{R}_{1}$ we obtain

$$
\theta_{1}=\arctan \left(\frac{\mathbf{T}_{\mathbf{1}} \cdot \mathbf{z}_{\mathbf{1}}}{\mathbf{T}_{\mathbf{1}} \cdot \mathbf{x}_{\mathbf{1}}}\right)
$$

As translation between these references is $h_{p} / 2$, we can therefore calculate the passage matrices between $\mathcal{R}_{1}$ and $\mathcal{R}_{2 a}$ or $\mathcal{R}_{2 b}$.

We can thus express $\mathbf{C}_{\mathbf{0}}(u)$ and $\mathbf{C}_{\mathbf{1}}(u)$ in the references $\mathcal{R}_{2 a}$ and $\mathcal{R}_{2 b}: \mathbf{C}_{\mathbf{0}} \mathbf{r}_{\mathbf{2}}(u)$ and $\mathbf{C}_{\mathbf{1}} \mathbf{r}_{\mathbf{2}} \mathbf{b}(u)$.

\subsection{Resolution}

The condition for tangency between the tool and the directrice $\mathbf{C}_{\mathbf{0}}(u)$ is translated by the unicity of the intersection between $\mathbf{C}_{\mathbf{0}}(u)$ and the ellipse $\mathbf{E}_{\mathbf{0}}(w)$ defined as the intersection of the tool and the plane $\left(\mathbf{P}_{\mathbf{0}}\right.$, $\left.\mathbf{x}_{\mathbf{2 a}}, \mathbf{y}_{\mathbf{2 a}}\right)$. By defining $\operatorname{tanEl}_{\mathbf{0}}(w)$ as the vector tangent to the ellipse $\mathbf{E}_{\mathbf{0}}(w)$ we can express the unicity of the intersection by the fact that $\operatorname{tanEll}_{\mathbf{0}}(w)$ and $\mathbf{C}_{\mathbf{0}}(u)$ have the same slope in reference $\mathcal{R}_{2 a}$, at the point of intersection.

We can calculate the coefficients for sign $\mu, \lambda_{0}$ and $\lambda_{1}$ such that:

If $\gamma>0, \mu=1$, if $\gamma<0, \mu=-1$ where $\gamma$ is the angle oriented $\left(\mathbf{z}_{1}, \mathbf{z}_{2}\right)$

If $\theta_{0}>0, \lambda_{0}=1$, if $\theta_{0}<0, \lambda_{0}=-1$ where $\theta_{0}$ is the angle oriented $\left(\mathbf{z}_{\mathbf{1}}, \mathbf{z}_{\mathbf{2}} \mathbf{a}\right)$

If $\theta_{1}>0, \lambda_{1}=1$, if $\theta_{1}<0, \lambda_{1}=-1$ where $\theta_{1}$ is the angle oriented $\left(\mathbf{z}_{1}, \mathbf{z}_{\mathbf{2}} \mathbf{b}\right)$

Further, we can postulate

$$
\gamma_{0}=|\gamma| \quad \text { whence } \quad \gamma=\mu \gamma_{0}
$$

The equation for $\operatorname{Ell}_{\mathbf{0}}(w)$ will be as follows, in the reference $\mathcal{R}_{2 a}$, (see Fig. 5):

$$
\mathbf{E l l}_{\mathbf{0}}(w)=\left(\begin{array}{c}
a_{0} \cos (w)+\mu L_{0} \\
R \sin (w)+R \\
0
\end{array}\right) \quad w \in[0,2 \pi]
$$

The vector $\operatorname{tanEl}_{\mathbf{0}}(w)$ tangent to $\operatorname{Ell}_{\mathbf{0}}(w)$ will be as follows, in the reference $\mathcal{R}_{2 a}$ (see Fig. 5):

$$
\begin{aligned}
& \operatorname{tanEll}_{\mathbf{0}}(w)=\frac{\frac{\partial \mathbf{E l}_{\mathbf{0}}(w)}{\partial w}}{\left\|\frac{\partial \mathbf{E} \mathbf{l}_{\mathbf{0}}(w)}{\partial w}\right\|}=\left(\begin{array}{c}
-a_{0} \sin (w) \\
R \cos (w) \\
0
\end{array}\right) \\
& w \in[0,2 \pi]
\end{aligned}
$$

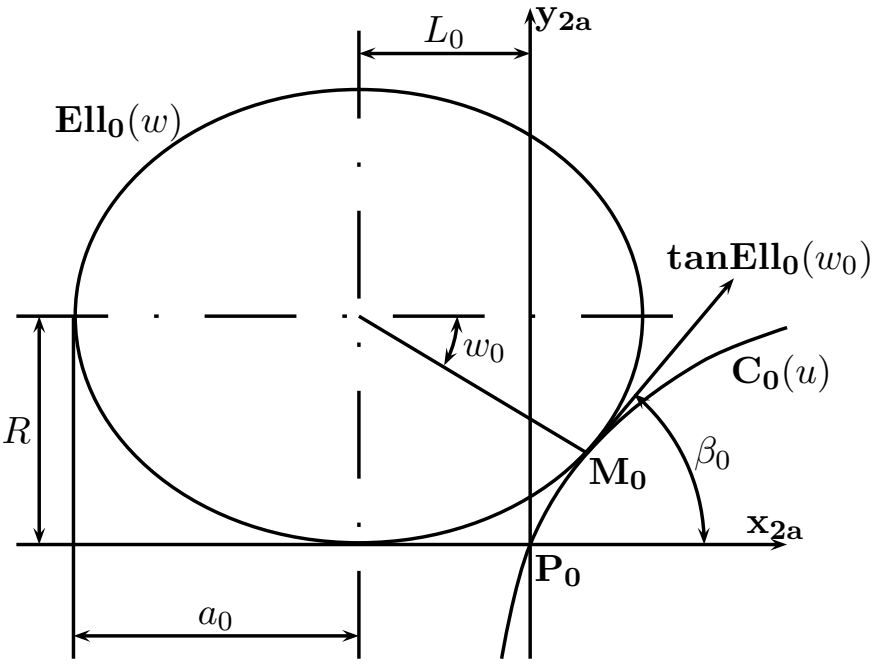

view along direction $\mathbf{z}_{\mathbf{2 a}}$

Figure 5: Ellipse $\mathbf{E l l}_{\mathbf{0}}(w)$

The vector $\mathbf{T}_{\mathbf{0}}(u)$ tangent to $\mathbf{C}_{\mathbf{0}}(u)$, in the reference $\mathcal{R}_{2 a}$ will be defined by

$$
\mathbf{T}_{\mathbf{0}}(u)=\frac{\frac{\partial \mathbf{C}_{\mathbf{0}}(u)}{\partial u}}{\left\|\frac{\partial \mathbf{C}_{\mathbf{0}}(u)}{\partial u}\right\|} \quad u \in[0,1]
$$

and its slope $\beta_{0}$ will be given by

$$
\beta_{0}=\arctan \left(\frac{\mathbf{T}_{\mathbf{0}} \cdot \mathbf{y}_{\mathbf{2}}}{\mathbf{T}_{\mathbf{0}} \cdot \mathbf{x}_{\mathbf{2}}}\right)
$$

Further, we shall obtain (see Fig. 4):

$$
\tan \left(\gamma_{0}\right)=\frac{L_{0} \cos \left(\lambda_{0} \theta_{0}\right)}{h_{0}-\mu \lambda_{0} L_{0} \sin \left(\lambda_{0} \theta_{0}\right)}
$$

whence:

$$
L_{0}=\frac{h_{0} \tan \left(\gamma_{0}\right)}{\cos \left(\lambda_{0} \theta_{0}\right)+\mu \lambda_{0} \tan \left(\gamma_{0}\right) \sin \left(\lambda_{0} \theta_{0}\right)}
$$

and (see Fig. 4):

$$
\cos \left(\lambda_{0} \theta_{0}-\mu \lambda_{0} \gamma_{0}\right)=\frac{R}{a_{0}}
$$

whence:

$$
a_{0}=\frac{R}{\cos \left(\lambda_{0}\left(\theta_{0}-\mu \gamma_{0}\right)\right)}
$$

Similarly, in the reference $\mathcal{R}_{2 b}$ we have: The equation for $\operatorname{Ell}_{\mathbf{1}}(w)$ :

$$
\mathbf{E l l}_{\mathbf{1}}(w)=\left(\begin{array}{c}
a_{1} \cos (w)-\mu L_{1} \\
R \sin (w)+R \\
0
\end{array}\right) \quad w \in[0,2 \pi]
$$


The vector $\tan \operatorname{Ell}_{\mathbf{1}}(w)$ tangent to $\operatorname{Ell}_{\mathbf{1}}(w)$ :

$$
\begin{aligned}
& \operatorname{tanEll}_{\mathbf{1}}(w)=\frac{\frac{\partial \mathbf{E l}_{1}(w)}{\partial(w)}}{\left\|\frac{\partial \mathbf{E l}_{\mathbf{1}}(w)}{\partial(w)}\right\|}=\left(\begin{array}{c}
-a_{1} \sin (w) \\
R \cos (w) \\
0
\end{array}\right) \\
& w \in[0,2 \pi]
\end{aligned}
$$

The vector $\mathbf{T}_{\mathbf{1}}(u)$ tangent to $\mathbf{C}_{\mathbf{1}}(u)$ :

$$
\mathbf{T}_{\mathbf{1}}(u)=\frac{\frac{\partial \mathbf{C}_{\mathbf{1}}(u)}{\partial u}}{\left\|\frac{\partial \mathbf{C}_{\mathbf{1}}(u)}{\partial u}\right\|} \quad u \in[0,1]
$$

whence its slope

$$
\beta_{1}=\arctan \left(\frac{\mathbf{T}_{\mathbf{1}} \cdot \mathbf{y}_{\mathbf{2 b}}}{\mathbf{T}_{\mathbf{1}} \cdot \mathbf{x}_{\mathbf{2}}}\right)
$$

In the same way as in $\mathcal{R}_{2 a}$ reference, we obtain:

$$
L_{1}=\frac{h_{1} \tan \left(\gamma_{0}\right)}{\cos \left(\lambda_{1} \theta_{1}\right)+\mu \lambda_{1} \tan \left(\gamma_{0}\right) \sin \left(\lambda_{1} \theta_{1}\right)}
$$

and:

$$
a_{1}=\frac{R}{\cos \left(\lambda_{1}\left(\theta_{1}-\mu \gamma_{0}\right)\right)}
$$

Using (4) and (5) in (1) and (2), we obtain the equations at the point of contact $\mathbf{M}_{\mathbf{0}}$ :

The intersection at $\mathbf{M}_{\mathbf{0}}$ will be translated by

$$
\mathbf{C}_{\mathbf{0}} \mathbf{r}_{\mathbf{2}}\left(u_{0}\right) \cdot \mathbf{x}_{\mathbf{2 a}}=\mathbf{E l l}_{\mathbf{0}}\left(w_{0}\right) \cdot \mathbf{x}_{\mathbf{2} \mathbf{a}}
$$

and

$$
\mathbf{C}_{\mathbf{0}} \mathbf{r}_{\mathbf{2}}\left(u_{0}\right) \cdot \mathbf{y}_{2 \mathbf{a}}=\mathbf{E l l}_{\mathbf{0}}\left(w_{0}\right) \cdot \mathbf{y}_{\mathbf{2} \mathbf{a}}
$$

That is,

$$
\begin{array}{r}
\mathbf{C}_{\mathbf{0}} \mathbf{r}_{\mathbf{2} \mathbf{a}}\left(u_{0}\right) \cdot \mathbf{x}_{\mathbf{2 a}}=\frac{R}{\cos \left(\lambda_{0}\left(\theta_{0}-\mu \gamma_{0}\right)\right)} \cos \left(w_{0}\right) \\
+\mu \frac{h_{0} \tan \left(\gamma_{0}\right)}{\cos \left(\lambda_{0} \theta_{0}\right)+\mu \lambda_{0} \tan \left(\gamma_{0}\right) \sin \left(\lambda_{0} \theta_{0}\right)}
\end{array}
$$

and

$$
\mathbf{C}_{\mathbf{0}} \mathbf{r}_{\mathbf{2} \mathbf{a}}\left(u_{0}\right) \cdot \mathbf{y}_{\mathbf{2} \mathbf{a}}=R \sin \left(w_{0}\right)+R
$$

The equality of slopes between $\operatorname{tanEl}_{\mathbf{0}}(w)$ and $\mathbf{C}_{\mathbf{0}} \mathbf{r}_{\mathbf{2}} \mathbf{a}(u)$ will be translated by

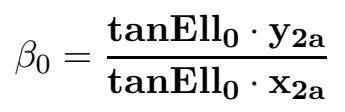

That is

$$
\beta_{0}=\frac{-\cos \left(\lambda_{0}\left(\theta_{0}-\mu \gamma_{0}\right)\right)}{\tan \left(w_{0}\right)}
$$

Similarly, we shall obtain the following in reference $\mathcal{R}_{2 b}$ :

$$
\begin{gathered}
\mathbf{C}_{\mathbf{1}} \mathbf{r}_{\mathbf{2} \mathbf{b}}\left(u_{1}\right) \cdot \mathbf{x}_{\mathbf{2} \mathbf{b}}=\frac{R}{\cos \left(\lambda_{1}\left(\theta_{1}-\mu \gamma_{0}\right)\right)} \cos \left(w_{1}\right) \\
-\mu \frac{h_{1} \tan \left(\gamma_{0}\right)}{\cos \left(\lambda_{1} \theta_{1}\right)+\mu \lambda_{1} \tan \left(\gamma_{0}\right) \sin \left(\lambda_{1} \theta_{1}\right)} \\
\mathbf{C}_{\mathbf{1}} \mathbf{r}_{\mathbf{2}} \mathbf{b}\left(u_{1}\right) \cdot \mathbf{y}_{\mathbf{2} \mathbf{b}}=R \sin \left(w_{1}\right)+R \\
\beta_{1}=\frac{-\cos \left(\lambda_{1}\left(\theta_{1}-\mu \gamma_{1}\right)\right)}{\tan \left(w_{1}\right)}
\end{gathered}
$$

Further, we know that

$$
h_{0}+h_{1}=h_{p}
$$

Whence a system of 7 equations with 7 unknown values $\left(u_{0}, u_{1}, w_{0}, w_{1}, h_{0}, h_{1}\right.$ and $\left.\gamma\right)$ that can be resolved numerically in a sufficiently short time.

\section{Error Calculation and Analysis}

\subsection{Error Calculation Methodology}

The errors on both sides of point $\mathbf{M}_{\mathbf{2}}$ are calculated separately.

Comment: the iteration increment between two successive rules will then be considered small enough for the two successive positions of the tool to be included within the envelope of tool positions. Otherwise, the error would need to be calculated in relation to this envelope, which would require an excessively long calculation time.

The calculation is carried out by postulating

$$
\mathbf{S}(u, v)=\mathbf{F}\left(s_{i}, t_{i}\right)+\varepsilon \mathbf{n}_{\mathbf{T}}\left(s_{i}, t_{i}\right)
$$

where $\mathbf{F}\left(s_{i}, t_{i}\right)$ is the point of the tool where the calculation is carried out, $s_{i}$ and $t_{i}$ are values for parameters at that point, $\mathbf{n}_{\mathbf{T}}\left(s_{i}, t_{i}\right)$ the unit vector perpendicular to the tool at that point, and $\varepsilon$ the value sought for. This equation can thus be reduced to a system of three equations and three unknown values: $u, v$ and $\varepsilon$. In this equation, $s_{i}$ and $t_{i}$ are taken between the limits of the parametric interval for calculation determined by the contact points. We can then choose the values for parameters at contact points $\mathbf{M}_{\mathbf{0}}, \mathbf{M}_{\mathbf{1}}$, and $\mathbf{M}_{\mathbf{2}}$ as limits for our calculation interval.

The resolution algorithm adopted will be a dichotomial algorithm with variation in the two parametric directions $s$ and $t$. 
Between $\mathbf{M}_{\mathbf{2}}$ and $\mathbf{M}_{\mathbf{0}}$, we take the following as our calculation interval:

$$
\begin{gathered}
s_{2}<s_{i}<s_{0} \\
t_{2}<t_{i}<t_{0}
\end{gathered}
$$

While between $\mathbf{M}_{\mathbf{2}}$ and $\mathbf{M}_{\mathbf{1}}$, we take the following as our calculation interval:

$$
\begin{gathered}
s_{1}<s_{i}<s_{2} \\
t_{1}<t_{i}<t_{2}
\end{gathered}
$$

\subsection{Analysis Principle}

Given that we cannot carry out an analytical error calculation, the error calculation study is made through a range of representative examples. For this purpose, we take into account the fact that the main parameters having an influence on error are:

- The tool radius $\mathrm{R}$.

- The angle $\alpha$ made by the two perpendiculars $\mathbf{N}_{\mathbf{0}}$ and $\mathbf{N}_{1}$.

- The length of the rule $h_{p}$.

We will study the specific influence of each parameter on the error made through tests carried out on a hyperbolic paraboloid, allowing us to change each parameter independently.

The hyperbolic paraboloid we studied was based on the two following directrices:

$$
\mathbf{C}_{\mathbf{0}}(u)=\left(\begin{array}{c}
k u \\
k \tan \left(\alpha_{0}\right)\left(u-\frac{1}{2}\right) \\
\frac{h_{p}}{2}
\end{array}\right)
$$

and

$$
\mathbf{C}_{\mathbf{1}}(u)=\left(\begin{array}{c}
k u \\
-k \tan \left(\alpha_{0}\right)\left(u-\frac{1}{2}\right) \\
\frac{-h_{p}}{2}
\end{array}\right)
$$

whence the parametered surface

$$
\mathbf{S}(u, v)=\left(\begin{array}{c}
k u \\
-\frac{1}{2} k \tan \left(\alpha_{0}\right)(4 u v-2 u-2 v+1) \\
\frac{1}{2} h_{p}-v h_{p}
\end{array}\right)
$$

where $k$ is a parameter for scale, we shall take $k=h_{p}$.

In order to validate this approach, we compared the error curves obtained with any ruled surface (that defined in (18), see Fig. 6) and a hyperbolic paraboloid (see Fig. 7). We can observe qualitatively similar curve shapes.

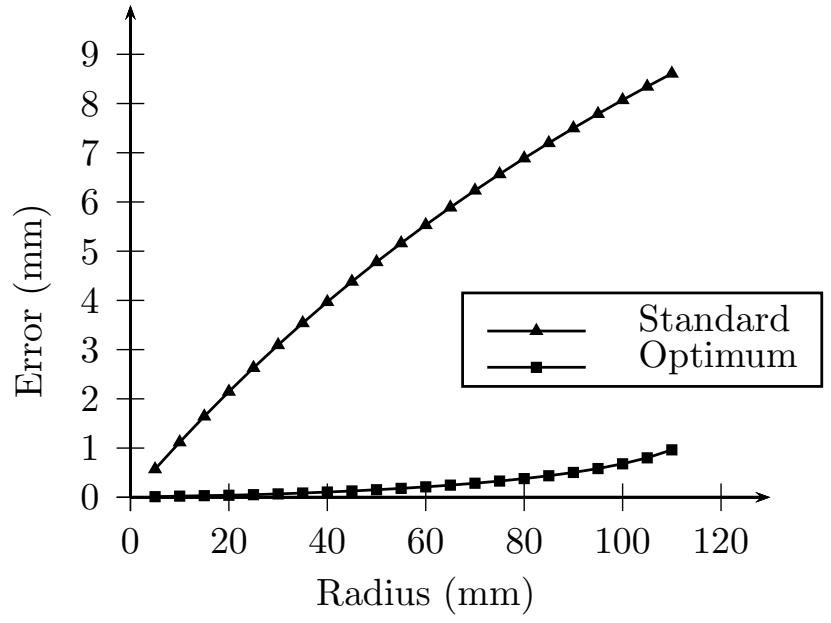

Figure 6: Error curves for any ruled surface

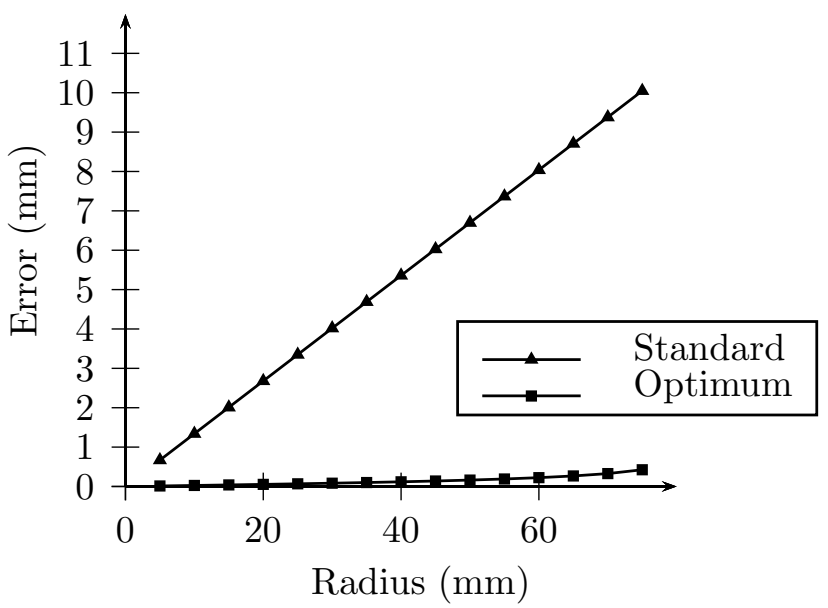

Figure 7: Error curves for a hyperbolic paraboloid $\left(\alpha=30^{\circ}, h_{p}=80 \mathrm{~mm}\right)$

\section{Results and Conclusions}

\subsection{Qualitative Analysis}

The setting we suggest for the tool would appear to allow for a considerable reduction in interference between the tool and the surface.

Further, the calculation time needed to determine setting parameters and error is no greater than a few seconds, which leads us to think that implementation in $\mathrm{CAD} / \mathrm{CAM}$ type software is feasible.

As an example, we carried out comparative analysis for any ruled surface: 

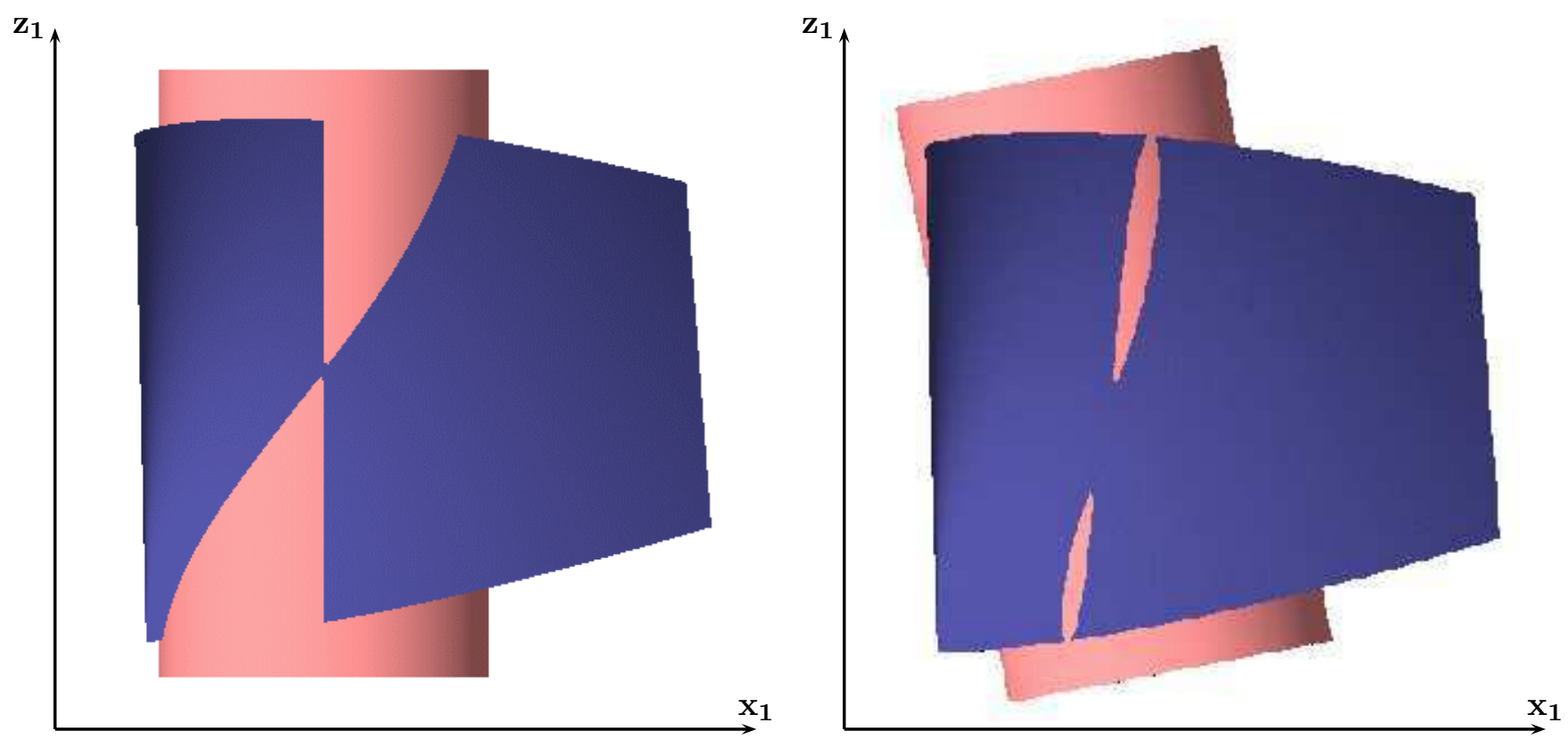

Figure 8: Error localisation

\subsection{Quantitative Analysis}

$\mathbf{S}(u, v)=\left(\begin{array}{c}30 u^{2}+10 u+30+20 v u^{2}-10 v u-10 v \\ 100 \sqrt{u}-50-100 v \sqrt{u}+90 v-60 v \sin \left(\frac{1}{2} \pi u\right) \\ 70-30 u^{2}-130 v+50 v u^{2}\end{array}\right)$

For this surface, we chose $u_{p}=0.4$ and $R=40$.

With standard setting with error distribution, $\max$ interference will be $\varepsilon=4.880 \mathrm{~mm}$.

With the optimum setting we suggest max interference will be $\varepsilon=0.181 \mathrm{~mm}$.

Figure 8 allows error localisation in each case to be appreciated qualitatively.

Figure 8 also allows another advantage of the suggested model to be highlighted, which is to respect the directrices of the ruled surface which can prove to be extremely useful in a large number of cases, particularly within the perspective of a circumferential machining method for any form of surface using piecewise approximation as proposed in [11]. We can thus envisage a side milling methodology for complex surfaces, which is particularly valid for convex and double curved surfaces, allowing for a considerable gain in time in relation to conventional end machining techniques.

Further, this model enabled us to develop a computation algorithm for the optimum tool radius for a given case. The major difficulty encountered was in optimising the algorithm to obtain this radius with a minimum number of iterations.

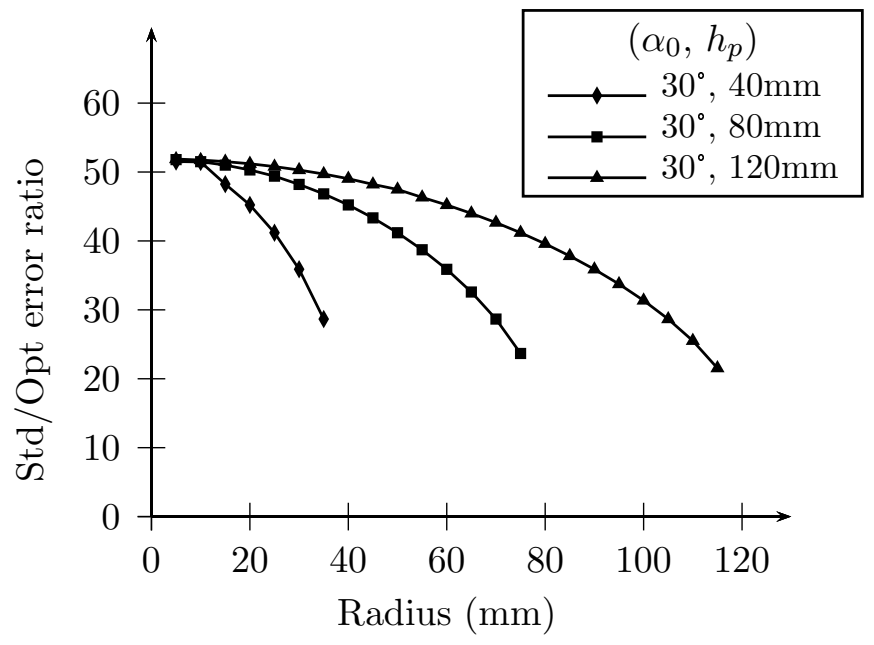

Figure 9: Performance ratings in relation to $h_{p}$.

We chose the ratio of error for standard setting over error for optimised setting as the indicator for performance of our setting as compared with standard positioning. Figures 9 and 10 show the variation of this indicator in relation to $R$ for different values of Oto on the one hand and hp o $\mathrm{n}$ the other hand.

All the results obtained hitherto show that the model we suggest allow errors to be reduced by a ratio of at least 10 to !.

Further, for the lowest performance indicator obtained (e o $=45)$, it can be observed that the gain is greater from a quantitative point of view. 


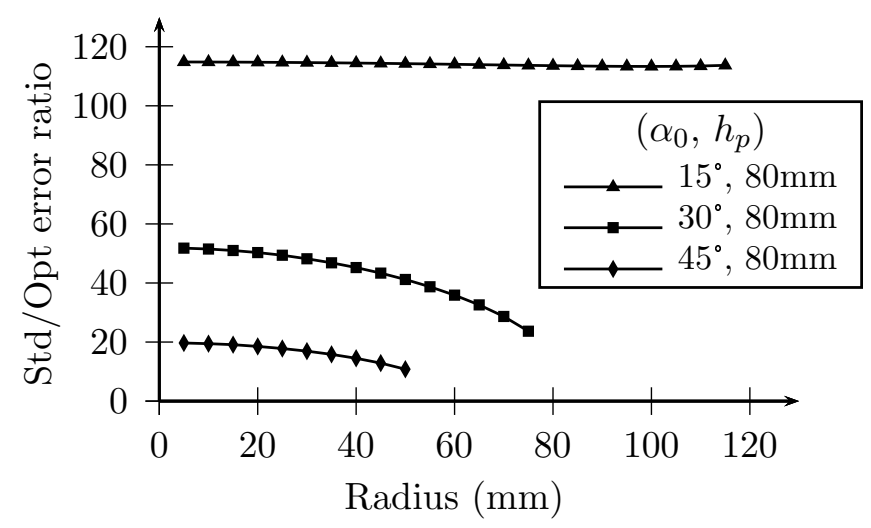

Figure 10: Performance ratings in relation to $\alpha_{0}$

In [7], Liu describes a method applied to the ruled surface defined from directrices:

$$
\mathbf{C}_{\mathbf{0}}(u)=\left(\begin{array}{c}
u \\
20.429 \\
0
\end{array}\right) \quad \text { and } \quad \mathbf{C}_{\mathbf{1}}(u)=\left(\begin{array}{c}
u \\
0.0382 u^{2} \\
33.995
\end{array}\right)
$$

for $0 \leqslant u \leqslant 23.014$ and $0 \leqslant v \leqslant 1$

The tool radius $R=100 \mathrm{~mm}$.

Using the model described by Liu, the theoretical error made in machining this surface with this tool radius is $\varepsilon= \pm 0.585 \mathrm{~mm}$.

Using the model we suggest, for the same radius $R=10 \mathrm{~mm}$, we obtained $\varepsilon=0.22 \mathrm{~mm}$, i.e. a performance ratio of $(0.585 \times 2) / 0.22=5.3$.

\section{References}

[1] K. Marciniak. Geometric Modelling for Numerically Controlled Machinig. Oxford University Press, 1991.

[2] B. K. Choi, J. W. Park, and C. S. Jun. Cutter location data optimization in 5-axis surface machining. Computer Aided Design, 25(6):377-386, 1993.

[3] Y.-S. Lee and T.-C. Chang. Machined surface error analysis for 5-axis machining. International Journal of Production Research, 34(1):111-135, 1996.

[4] S. X. Li and R. B. Jerard. 5-axis machining of sculptured surfaces with a flat-end cutter. Computer Aided Design, 26(3):165-178, 1994.
[5] W. Rubio, P. Lagarrigue, G. Dessein, and F. Pastor. Calculation of Tool Paths for a Torus Mill on Free-Form Surfaces Five-Axis Machines with Detection and Elimination of Interference. The International Journal of Advanced Manufacturing Technology, 14:13-20, 1998.

[6] W. Rubio. Génération de trajectoires du centre de l'outil pour l'usinage de surfaces complexes sur machines à trois et cinq axes. Thèse de doctorat, Université Paul Sabatier, Toulouse III, 1993.

[7] X. W. Liu. Five-Axis NC cylindrical milling of sculptured surfaces. Computer-Aided Design, 27(12):887-894, 1995.

[8] M. D. Carmo. Differential Geometry of Curves and Surfaces. Prentice-Hall, 1976.

[9] D. Qiulin and B. J. Davies. Surface Engineering Geometry for Computer Aided Design and Manufacture. Ellis Horwood Ltd., 1987.

[10] I. D. Faux and M. J. Pratt. Computational Geometry for Design and Manufacture. Mathematics and its applications. Ellis Horwood Ltd., 4ème edition, 1985.

[11] G. Elber and F. Russ. 5 Axis free-form surface milling using piecewise ruled surface approximation. ASME Journal of Engineering for Industry, 119(3):383-387, august 1997. 